\title{
HIV-associated Cryptococcal Meningitis: a Review of Novel Short-Course and Oral Therapies
}

\author{
Letumile R. Moeng, $\mathrm{MBBS}^{1,{ }^{*}} \odot$ \\ James Milburn, MBChB $B^{2,3}$ \\ Joseph N. Jarvis, MBBS, MSc, PhD 2,3 \\ David S. Lawrence, MBChB, MSc $c^{2,3}$
}

\author{
Address \\ ${ }^{*}, 1$ Center for Infectious Disease Management and Research, Department of Inter- \\ nal Medicine, Howard University Hospital, Washington, DC, USA \\ Email: Imoeng@huhosp.org \\ ${ }^{2}$ Clinical Research Department, Faculty of Infectious and Tropical Diseases, Lon- \\ don School of Hygiene and Tropical Medicine, London, UK \\ ${ }^{3}$ Botswana Harvard AIDS Institute Partnership, Gaborone, Botswana
}

Published online: 4 November 2020

(C) The Author(s) 2020

This article is part of the Topical Collections on HIV Medicine

Keywords HIV • Cryptococcal meningitis · Fluconazole · Flucytosine · Amphotericin

\section{Abstract}

Purpose of review HIV-associated cryptococcal meningitis remains a significant public health problem in parts of Africa and Asia and a major cause of AIDS-related mortality, accounting for $15 \%$ of all AIDS-related deaths worldwide. Cryptococcal meningitis is uniformly fatal if untreated, and access to antifungal therapy in regions with the highest burden is often limited. Outcomes with fluconazole monotherapy are poor, and induction treatment with amphotericin B and high-dose fluconazole for 2 weeks is associated with significant drug-related toxicities and prolonged hospital admissions. This review focuses on the potential of novel short-course and oral combination therapies for cryptococcal meningitis.

Recent findings Recent clinical trials have shown that shorter courses of amphotericin, if paired with oral flucytosine, rather than fluconazole, can achieve non-inferior mortality outcomes. In addition, an oral combination of fluconazole and flucytosine is a potential alternative. Liposomal amphotericin B may further simplify treatment; it is associated with fewer drug-related toxicities, and a recent phase II randomised controlled trial demonstrated that a single, high dose of liposomal amphotericin is non-inferior to 14 standard daily doses at clearing Cryptococcus from cerebrospinal fluid. This has been taken forward to an ongoing phase III, clinical endpoint study. 
Summary The incidence and mortality associated with cryptococcal meningitis is still unacceptably high. There is evidence supporting the use of short-course amphotericin B and oral combination antifungal treatment regimens for cryptococcal meningitis (CM). Ongoing research into short-course, high-dose treatment with liposomal amphotericin may also help reduce the impact of this devastating disease.

\section{Introduction}

HIV-associated cryptococcal meningitis (CM) remains a significant public health problem and a major cause of AIDS-related mortality, despite increasing access to antiretroviral therapy (ART) $[1,2]$. There are an estimated 220,000 cases each year, with the greatest burden of disease found within resource-limited, high HIV prevalence settings. Across sub-Saharan Africa, CM remains the most common cause of meningitis in adults living with HIV [3]. As ART programmes were introduced, the number of new cases fell, but in recent years, and despite widened ART rollout, this number has plateaued with CM being responsible for more than 180,000 annual deaths and $15 \%$ of all AIDS-related mortality globally $[4 \bullet]$.
This review will focus on new approaches to the treatment of $\mathrm{CM}$, with a specific focus on the potential of short-course or oral combination antifungal treatment regimens in low- and middle-income countries (LMICs). We outline the limitations of the currently available treatment options, summarise recently published research that has changed treatment guidelines, and describe ongoing and future studies that may further inform this approach. We then contextualise these data and discuss current barriers to implementation that need to be overcome, particularly regarding access to medications in resource-limited settings.

\section{The current treatment paradigm}

Cryptococcal meningitis is uniformly fatal if left untreated [4•]. Treatment of $\mathrm{CM}$ is challenging, requiring intensive antifungal therapy, the identification and management of drug-related toxicities, strict control of raised intracranial pressure, and the safe promotion of immune recovery with ART. The median CD4 count of individuals diagnosed with $\mathrm{CM}$ is approximately 30 cells $/ \mu \mathrm{L}[5 \bullet \bullet]$, and therefore, the management of these patients also involves the diagnosis and treatment of other complications of advanced HIV disease.

The unacceptably high mortality associated with $\mathrm{CM}$ is driven, at least in part, by the limited availability of effective antifungal medication in LMICs. The most widely used oral antifungal treatment is fluconazole which is affordable, widely available (often through donation), and has a good safety profile. When given in high doses (800-1200 mg per day) as monotherapy for $\mathrm{CM}$ in a clinical trial setting, fluconazole is associated with a 10-week mortality of 50$60 \%[6,7]$. The limited efficacy of fluconazole when used in monotherapy is attributed to its predominantly fungistatic activity, inhibiting growth of Cryptococcus to achieve a therapeutic effect, in contrast to other agents such as amphotericin which are fungicidal. Fluconazole remains predominantly fungistatic even at high doses; when maximally tolerated doses are given, clinical failure rates remain high in comparison to other regimens [8]. In addition, 
fluconazole susceptibility in Cryptococcus species appears to be decreasing. A systematic review in 2019 identified a rise in the median fluconazole minimum inhibitory concentration (MIC) from $4 \mu \mathrm{g} / \mathrm{mL}$ between 2000 and 2012 to $8 \mu \mathrm{g} /$ mL globally between 2014 and 2018 [9]. Similar findings were observed in South Africa where the MICs of prospectively collected cryptococcal isolates demonstrated a doubling of the geometric mean MIC between 2007 and 2008 and 2017 from 2.08 to $4.11 \mu \mathrm{g} / \mathrm{mL}$ [10]. Furthermore, a recent study demonstrated that even in settings where primary genetic fluconazole resistance is rare, poor fungal clearance occurs due to the phenomenon of heteroresistance: small numbers of resistant subpopulations existing at levels low enough not to exert a significant effect on the MIC but, in the presence of fluconazole alone, susceptible subpopulations die and resistant clones dominate [11].

Given the marked limitations of fluconazole monotherapy, most international guidelines for the management of $\mathrm{CM}$ in resource-limited settings have recommended the addition of amphotericin $\mathrm{B}$ deoxycholate which, when administered for 2 weeks alongside high-dose fluconazole, is associated with roughly $30-40 \%$ mortality at 10 weeks $[5 \bullet \bullet]$ in clinical trial settings. However, amphotericin B is notoriously toxic, and induction treatment for 2 weeks is commonly associated with thrombophlebitis, as well as an average drop in haemoglobin of $2.3 \mathrm{~g} / \mathrm{dL}$ and an increase in creatinine of 73\% [12] (Fig. 1). These toxicities require regular monitoring which may be difficult in settings with limited healthcare workers and laboratory capacity. When comparing outcome data from clinical trials with those collected through observational
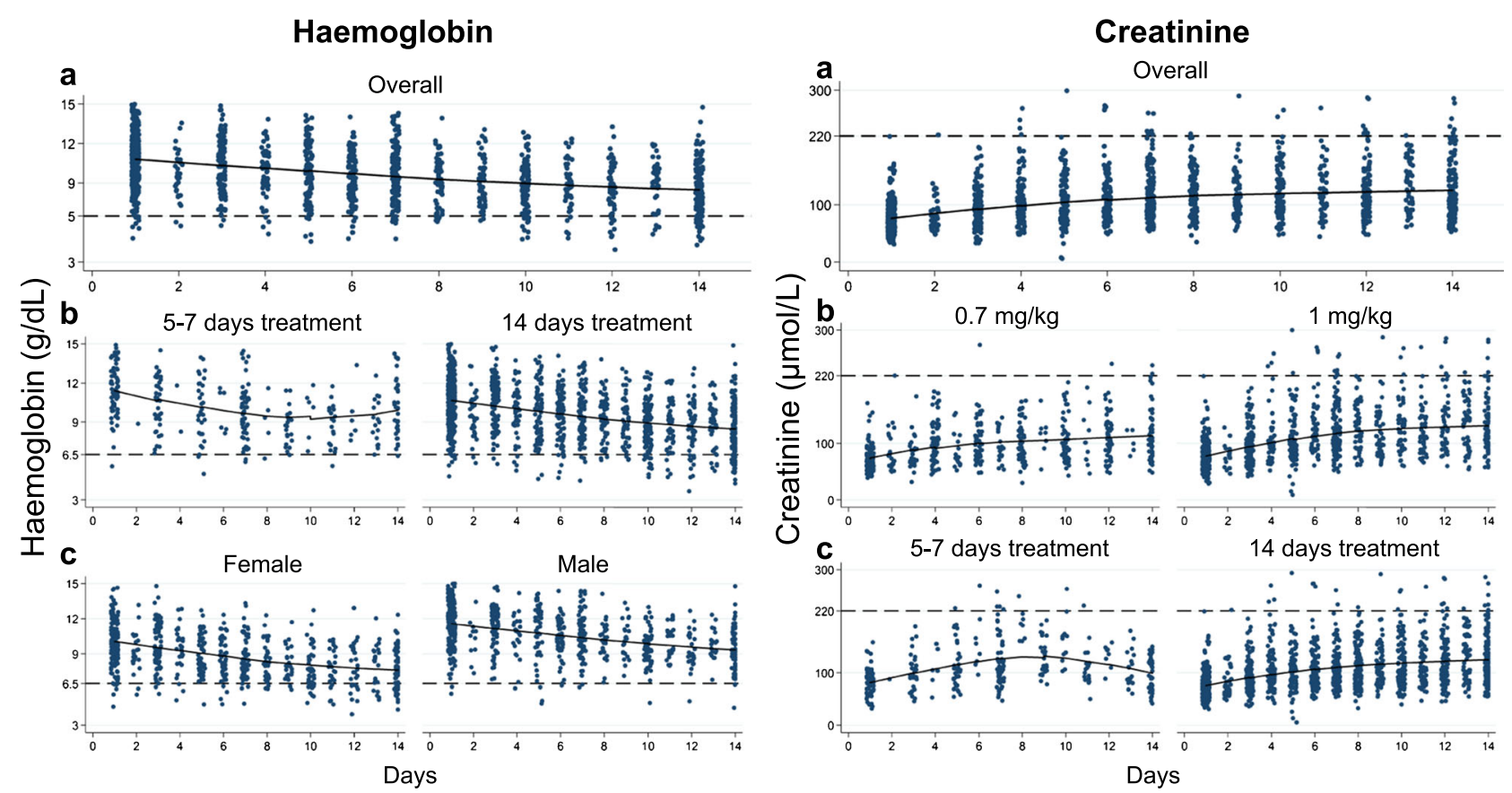

Fig. 1. Individual data points and fitted locally weighted scatterplot smoothing curves for haemoglobin (left) and creatinine (right) values over the first 14 days of antifungal therapy. (a) All patient receiving 14 days of amphotericin B based induction therapy. (b) Plot by amphotericin B duration (short-course versus standard treatment). (c) Plot by sex. The broken lines indicate an anaemia threshold of $6.5 \mathrm{~g} / \mathrm{dL}$ (division of AIDS grade IV adverse event) and a creatinine of $220 \mu \mathrm{mol} / \mathrm{L}$, indicating nephrotoxicity [12]. 
studies, these challenges appear to translate to higher mortality rates in routine care. Data collected from the national death registry in Botswana between 2012 and 2014, where amphotericin B and fluconazole was the standard of care, identified a 10 -week mortality of 50\% [13]. In comparison, randomised controlled trials of this regimen conducted in Vietnam and across multiple sites in Africa have demonstrated lower mortality rates of $30-40 \%[5,14]$. Whilst selection bias may influence the overall mortality rates in some clinical trials, closer monitoring for toxicities and enhanced clinical care likely confer a significant survival benefit.

The combination of 2 weeks of intravenous treatment alongside the associated toxicity means that managing a CM patient with this regimen involves a minimum hospital stay of 14 days and regular blood tests. As a consequence, in Uganda, the hospitalisation costs are approximately USD 560 [15]. In Zimbabwe, costs are approximately USD 800-1000 [16], and in South Africa, the cost is estimated at USD 2453 [17]. Finally, amphotericin B must be refrigerated, and the maintenance of a cold chain poses logistical challenges in resourcelimited settings.

\section{Shorter-course amphotericin-based treatment}

The major challenges of managing patients with two-week courses of amphotericin B in low-resource settings have prompted investigation into whether shorter courses could provide similar antifungal effects and reduce the associated cost and toxicity. Data from animal models have paved the way for future clinical trials. In particular, a 2013 study using murine and rabbit models showed that 3 days of amphotericin B as induction therapy was as effective as daily therapy for 14 days. These animal data also showed a favourable pharmacokinetic profile of the abbreviated amphotericin B regimen, with sustained antifungal activity and persistence in the CSF (Fig. 2) [18•].

Two phase II clinical studies, one conducted in Uganda [19] and another in Malawi [7], both found participants treated with shorter courses of amphotericin B had good clearance of Cryptococcus from the brain and favourable side-effect profiles. The Ugandan study enrolled $30 \mathrm{HIV}$-infected patients with first-episode $\mathrm{CM}$ and treated them for 5 days with amphotericin $\mathrm{B}$ alongside 2 weeks of high-dose fluconazole. Repeat lumbar punctures were performed on days 3, 7, and 14 to establish the early fungicidal activity (EFA), a calculation of the mean rate of clearance of Cryptococcus from the CSF which correlates with the clinical outcome. Although the study was underpowered to detect any difference in mortality, it demonstrated mono-exponential fungal clearance over the observed 14 days with the calculated EFA being the same at 7 $(-0.31 \pm 0.14 \log \mathrm{CFU} /$ day $)$ and 14 days $(-0.30 \pm 0.11 \log \mathrm{CFU} /$ day $)$, thereby supporting the animal model data showing persistent antifungal effect after cessation of amphotericin B. The Malawian study, through a two-step process, randomised patients to one of four treatment arms: either 2 weeks of fluconazole monotherapy with or without amphotericin for 7 days or 2 weeks of fluconazole and flucytosine with or without amphotericin for 7 days. Similar to the Ugandan study, there was no reduction in EFA in the second week of treatment after the course of amphotericin B had been completed. In addition, when compared with historical cohorts treated with 2 weeks of amphotericin B, 

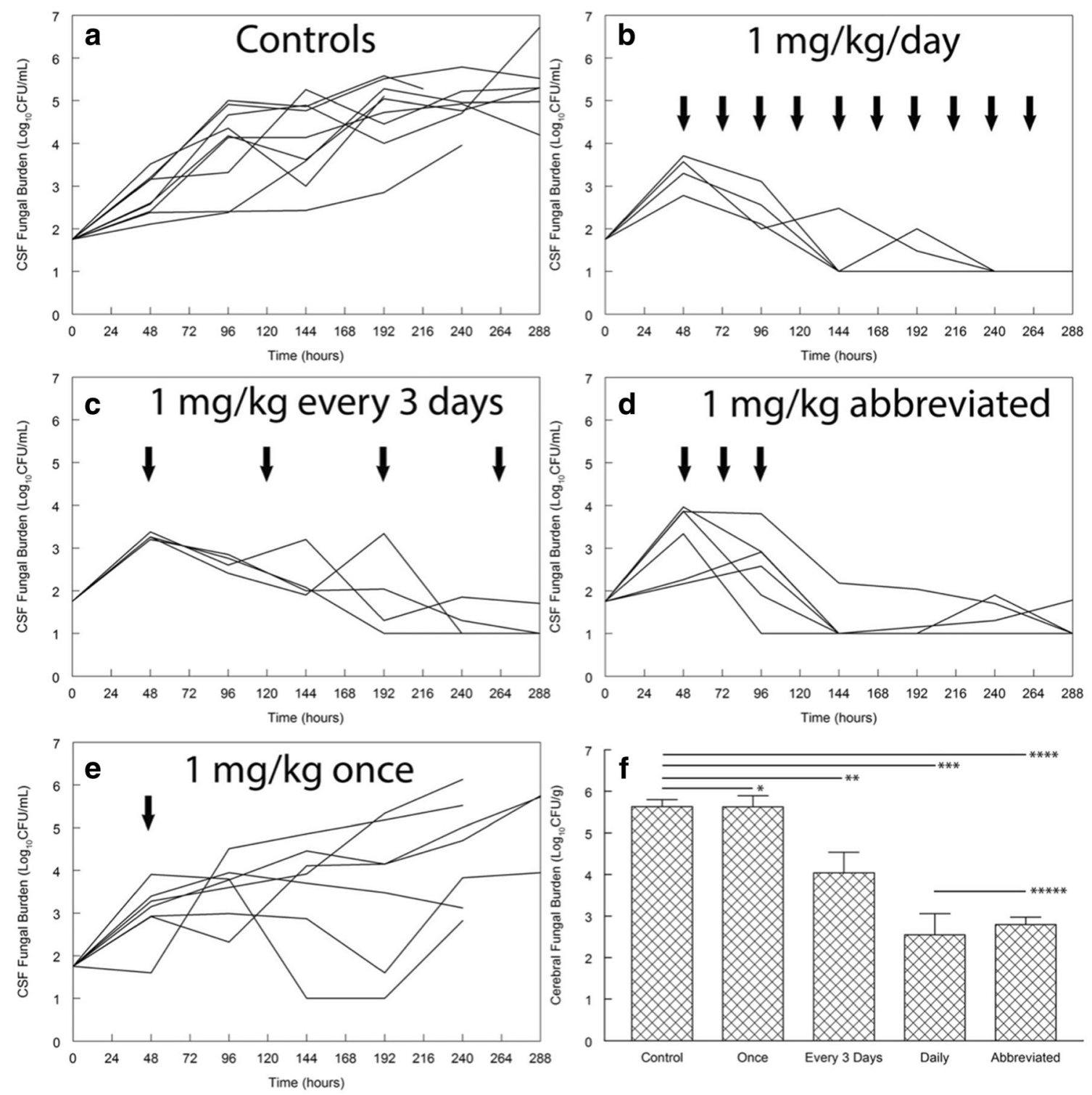

Fig. 2. Effects of various regimens of amphotericin B for experimental cryptococcal meningoencephalitis in rabbits. Amphotericin $B$ at $1 \mathrm{mg} / \mathrm{kg}$ was administered daily (b), every third day (c), as an abbreviated regimen for 3 consecutive days (d), or once (e). The solid arrows show the times of drug administration. The data in panels (a) to (e) represent the time course of infection in the CSF. Each line represents the fungal burden in an individual rabbit. Panel (f) shows the fungal density in the cerebrum at the end of the experiment. Each bar represents the mean fungal density \pm the standard error of the mean. There is no statistically significant difference in cerebral fungal density between rabbits that received daily therapy and those that received an abbreviated regimen of $1 \mathrm{mg} / \mathrm{kg}$ at 48,72 , and $96 \mathrm{~h}$ after inoculation. ${ }^{*}, p=1.00$ (not significant); ${ }^{* *}, p=0.006 ;{ }^{* * *}, p<0.001 ; * * * *, p<0.001 ;{ }^{* * * *}$, $p=1.00$ (not significant) [18•].

both of these studies found similar rates of EFA. The triple-combination treatment arm in the Malawian study, containing fluconazole, amphotericin $\mathrm{B}$, and flucytosine, had a significantly higher EFA $(-0.50 \pm 0.15 \log$ CFU/day $)$ than the other treatment arms (amphotericin and fluconazole $-0.38 \pm 0.19 \log \mathrm{CFU} /$ 
day; fluconazole and flucytosine $-0.28 \pm 0.17 \log$ CFU/day) supporting the rationale for phase III trials exploring both shorter-course amphotericin B treatment and the role of flucytosine.

\section{Flucytosine and the potential for oral combination therapy}

In addition to the potential benefits of shorter-course amphotericin-based regimens, the antifungal flucytosine has been tested both as an alternative and in addition to fluconazole. Flucytosine's effectiveness in combination therapy was demonstrated in a 1997 trial showing flucytosine to increase the rate of CSF sterilisation. In this study, $60 \%$ of patients receiving amphotericin $\mathrm{B}$ and flucytosine had negative CSF cultures at 2 weeks compared to $51 \%$ of patients treated with amphotericin alone [20]. Subsequent trials reiterated this and also highlighted flucytosine as superior to fluconazole when used in combination with amphotericin B. In a randomised controlled trial that recruited 299 participants in Vietnam, combination therapy with flucytosine was shown to confer a survival benefit at 10 weeks when compared to amphotericin alone (30\% versus $44 \%$; HR $0.61 ; 95 \%$ CI $0.39-0.97 ; p=0.04$ ). Despite a lower absolute mortality rate, there was no statistically significant difference in mortality when this arm was compared to amphotericin B given with fluconazole (30\% versus 33\%; HR 0.87; 95\% CI 0.53-1.42; $p=0.57$ ) [14].

In the same Malawian study discussed above, flucytosine and fluconazole combination therapy had a higher EFA than fluconazole alone [7]. A second study in Malawi also found this effect on EFA in addition to improved mortality at $2(10 \%$ versus $37 \%$; HR $0.24 ; 95 \%$ CI $0.05-1.16 ; p=0.05)$ and 10 weeks ( $43 \%$ versus 58\%; HR 0.59; 95\% CI $0.25-1.44 ; p=0.25$ ) [21]. One benefit of an oral combination antifungal treatment regimen is that it would not require any daily intravenous infusion which would eliminate the risk of treatmentassociated thrombophlebitis. An oral treatment could also potentially reduce the length of hospital admission, enabling milder or asymptomatic cases to continue induction therapy at home and therefore reducing the costs associated with CM. It is unlikely that this approach would ever obviate the need for hospital admission as CM patients are often very unwell and can deteriorate rapidly.

A highly influential study in this field was the Antifungal Combinations for Treatment of Cryptococcal Meningitis in Africa (ACTA) phase III randomised controlled trial. This clinical endpoint trial recruited participants in southern, eastern, and central Africa. HIV-infected patients with first-episode CM were randomised to one of five arms: 2 weeks of amphotericin $(1 \mathrm{mg} / \mathrm{kg} /$ day $)$ with either fluconazole (1200 mg/day) or flucytosine $(100 \mathrm{mg} / \mathrm{kg} /$ day $), 1$ week of amphotericin with either fluconazole or flucytosine, or oral combination therapy with flucytosine and fluconazole. The trial recruited a total of 721 participants, and a pooled analysis of mortality at 10 weeks identified that, irrespective of partner drug, treatment for 7 days with amphotericin B was non-inferior to treatment for 14 days (HR 0.89; 95\% CI 0.66-1.21) (Fig. 3). Those randomised to the arm receiving 7 days of amphotericin plus flucytosine 
A

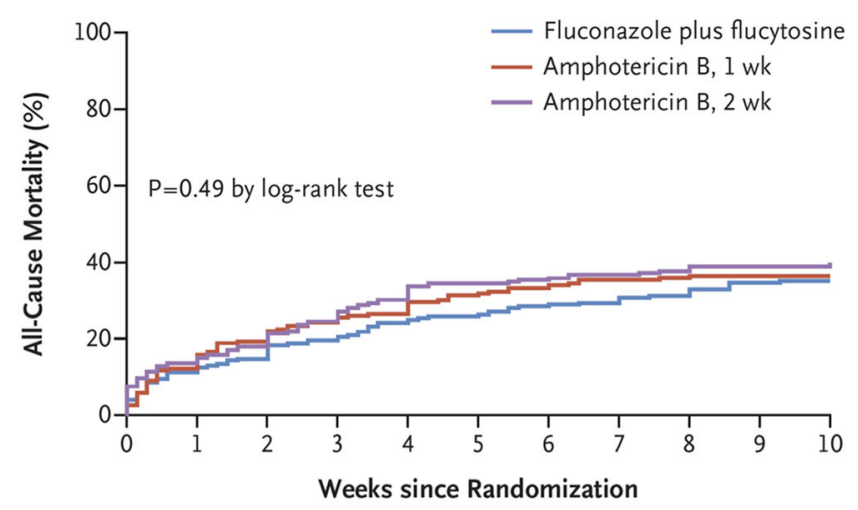

No. at Risk

$\begin{array}{llllllllllll}\text { Fluconazole plus flucytosine } & 225 & 200 & 192 & 181 & 171 & 167 & 161 & 159 & 155 & 147 & 144\end{array}$

$\begin{array}{llllllllllllll}\text { Amphotericin B, 1 wk } & 224 & 196 & 180 & 169 & 164 & 152 & 148 & 143 & 142 & 141 & 139\end{array}$

$\begin{array}{llllllllllll}\text { Amphotericin B, 2 wk } & 229 & 198 & 188 & 173 & 160 & 150 & 147 & 144 & 142 & 139 & 136\end{array}$

Fig. 3. Kaplan-Meier curves from the ACTA study illustrating the all-cause mortality at 10 weeks according to treatment strategy. The trial recruited a total of 721 participants, and mortality in the oral regimen, 1-week amphotericin B, and 2-week amphotericin B groups was $35.1 \%, 36.2 \%$, and $39.7 \%$ respectively. Irrespective of the partner drug, treatment with 7 days of amphotericin $B$ was non-inferior to treatment for 14 days (HR 0.89; 95\% CI 0.66-1.21) [5••].

experienced the lowest 10 -week mortality rate (24.2\%) when compared with all other regimes, including 2 weeks of amphotericin plus flucytosine (HR 0.56; 95\% CI; $0.35-0.91 ; p=0.001$ ).

As a partner drug to amphotericin B, flucytosine performed better than fluconazole (HR for death at 10 weeks with flucytosine vs. fluconazole 0.62; $95 \%$ CI $0.45-0.84 ; p=0.002$ ). All the flucytosine-containing regimens performed better, with the data showing that fluconazole as a partner drug to amphotericin B was associated with the highest 10 -week mortality: $48.6 \%$ when given for 1 week (indicating that flucytosine is essential if short courses of amphotericin are used) and $41.3 \%$ when given for 2 weeks of amphotericin B. Despite having the slowest rate of clearance of cryptococcus from the CSF, the oral combination arm was more effective in averting mortality than both of the amphotericin $\mathrm{B}$ and fluconazole arms, and was the second best performing arm with a 10 -week mortality rate of 35\% (95\% CI 28.9-41.3\%). This amphotericin-sparing regimen was also associated with the smallest median change in both haemoglobin $(-0.4 \mathrm{~g} / \mathrm{dL}$ IQR $-1.0-0.4)$ and creatinine (0 $\mu \mathrm{mol} / \mathrm{L} \mathrm{IQR} \mathrm{-} \mathrm{8.8-13.0).}$

Following the publication of the ACTA trial, a Cochrane review of treatment for CM [22] analysed 13 clinical trials, most of which were conducted in resource-limited settings. The review concluded that 1 week of amphotericin B with flucytosine probably resulted in a lower risk of death within 10 weeks, with shorter duration of amphotericin B therapy being associated with a lower risk of life-threatening toxicities. Furthermore, the review highlighted that combination oral therapy with flucytosine and fluconazole had a similar risk of death compared to the traditional 2 weeks of amphotericin B and flucytosine. Based on these findings, the World Health Organization updated their guidance on the management of $\mathrm{CM}$ in a resource-limited setting to recommend 1 week of amphotericin B with flucytosine as a first-line treatment in LMICs [23•]. In 
addition, where amphotericin B is not available, the oral combination therapy is recommended as a second-line regimen.

\section{The role of liposomal amphotericin}

The use of liposomal amphotericin (L-AmB, AmBisome, Gilead Sciences) has the potential to further improve outcomes in the management of CM. Despite being the mainstay of treatment in high-income settings [24, 25], L-AmB has not been widely used in LMICs due to high costs. L-AmB is however well-suited to short-course treatment of CM because high doses can be safely administered owing to lower rates of drug toxicity than amphotericin $\mathrm{B}$, and because the drug has a long half-life in the brain $[26,27]$. Animal model studies have shown efficacy with higher doses of LAmB $(10-20 \mathrm{mg} / \mathrm{kg} / \mathrm{day})[28,29]$. Studies to determine the shortest effective period of induction therapy with $\mathrm{L}-\mathrm{AmB}$ in murine and rabbit models used high-performance liquid chromatography to measure plasma and brain concentrations of L-AmB, finding a dose-dependent decline in fungal burden in the brain of mice, with a near-maximal efficacy achieved with LAmB at $10-20 \mathrm{mg} / \mathrm{kg} /$ day. Interestingly, the pharmacodynamics of a single dose of $20 \mathrm{mg} / \mathrm{kg}$ was the same as that of $20 \mathrm{mg} / \mathrm{kg} /$ day given for 2 weeks [29] (Fig. 4).

In addition, multiple human studies have also demonstrated safety when administering higher doses of L-AmB for the treatment of visceral leishmaniasis and invasive fungal infections in cancer patients [30]. A randomised controlled trial conducted in India found that a higher dose $\mathrm{L}-\mathrm{AmB}(10 \mathrm{mg} / \mathrm{kg})$ for the treatment of visceral leishmaniasis was not associated with an increase in adverse effects when compared with

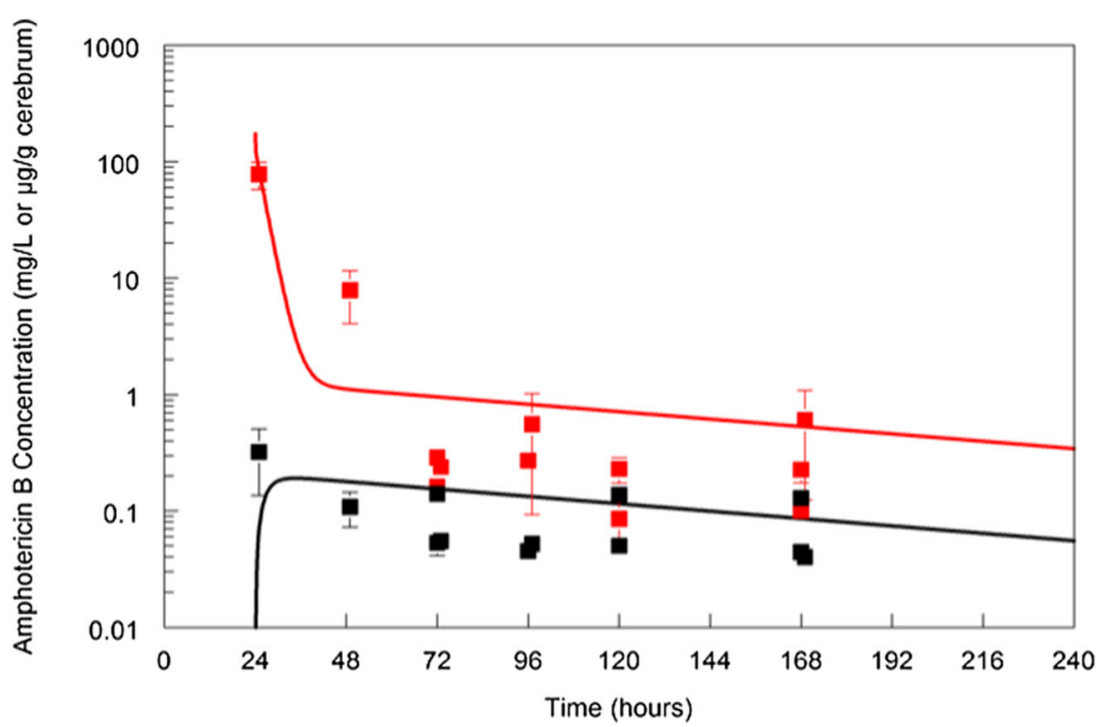

Fig. 4. The pharmacokinetics of liposomal amphotericin B in murine plasma (red) and cerebrum (black) in cohorts of mice infected with Cryptococcus neoformans receiving $20 \mathrm{mg} / \mathrm{kg}$ single-dose intravenously. The terminal half-life in the plasma and cerebrum is circa $133 \mathrm{~h}[28 \bullet]$. 
conventional therapy [31]. In this trial, a single dose of L-AmB $(10 \mathrm{mg} / \mathrm{kg})$ was compared with amphotericin B $(1 \mathrm{mg} / \mathrm{kg}$ administered every other day for 29 days). The study demonstrated similar cure rates of $95.7 \%$ (95\% CI 93.4-97.9) with L-AmB and 96.3\% (95\% CI 92.6-99.9) with conventional amphotericin B. A similar dose has also been used in studies of stem cell and liver transplant patients for antifungal prophylaxis, and both studies showed the dose was well-tolerated [32, 33]. The potential benefits of moving from daily intravenous amphotericin B to a single dose of L$A m B$ include fewer drug-induced toxicities, a shorter hospital stay, and a reduction in associated costs. In addition, L-AmB can be stored at an ambient temperature which would simplify distribution and storage of medication.

\section{The AMBITION study}

The AMBIsome Therapy Induction OptimisatiON (AMBITION) phase II trial was an open-label randomised non-inferiority trial to compare different short-course L-AmB regimens for the treatment of HIV-

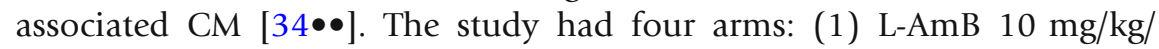
day on day 1 (single dose), (2) L-AmB $10 \mathrm{mg} / \mathrm{kg} /$ day on day 1 and $5 \mathrm{mg} / \mathrm{kg} /$ day on day 3 (2 doses), (3) L-AmB $10 \mathrm{mg} / \mathrm{kg} / \mathrm{day}$ on day 1 and $5 \mathrm{mg} / \mathrm{kg} /$ day on days 3 and 7 (3 doses), and (4) L-AmB $3 \mathrm{mg} / \mathrm{kg} /$ day for 14 days (control). All patients also received oral fluconazole $1200 \mathrm{mg} /$ day for 14 days. The primary endpoint was EFA and a total of 80 participants were recruited from sites in Botswana and Tanzania. The study found that the EFA in all short-course regimens was comparable to, or greater than, the control at the pre-defined non-inferiority margin. There was no evidence for any dose-response effect with additional doses. There were only 10 grade 3 and two grade 4 serious adverse events that were attributed to study drugs. These included one grade 3 and one grade 4 anaemia which compared favourably to historical data from 368 patients treated in Africa with amphotericin B where 33\% of patients reported a grade 3 or 4 anaemia [12]. Across all arms, the median drop in haemoglobin over the 2-week induction period was $0.9 \mathrm{~g} / \mathrm{dL}$ compared to a median drop of $2.3 \mathrm{~g} / \mathrm{dL}$ observed with amphotericin B [12]. Similarly, the percentage increase of baseline creatinine was $14 \%$ compared to $73 \%$ with amphotericin B (Fig. 5). Finally, the overall 10-week mortality was $29 \%$ which compared very favourably with mortality rates recorded elsewhere in the literature [14, 35-37].

The single, high-dose regimen has been taken forward to a clinical endpoint phase III trial which is currently recruiting participants from eight hospitals across five countries in southern and eastern Africa [16]. The AMBITION phase III trial will recruit 850 individuals with HIV-associated cryptococcal meningitis and randomise them $1: 1$ to either (1) single-dose L-AmB $(10 \mathrm{mg} / \mathrm{kg})$ given alongside 14 days of fluconazole $(1200 \mathrm{mg} /$ day $)$ and flucytosine $(100 \mathrm{mg} / \mathrm{kg} /$ day); or (2) 7 days of amphotericin B (1 mg/kg/day) given alongside 7 days of flucytosine $(100 \mathrm{mg} / \mathrm{kg} /$ day $)$ and followed by 7 days of fluconazole $(1200 \mathrm{mg} /$ day). The primary endpoint is all-cause mortality at 10 weeks. The trial 

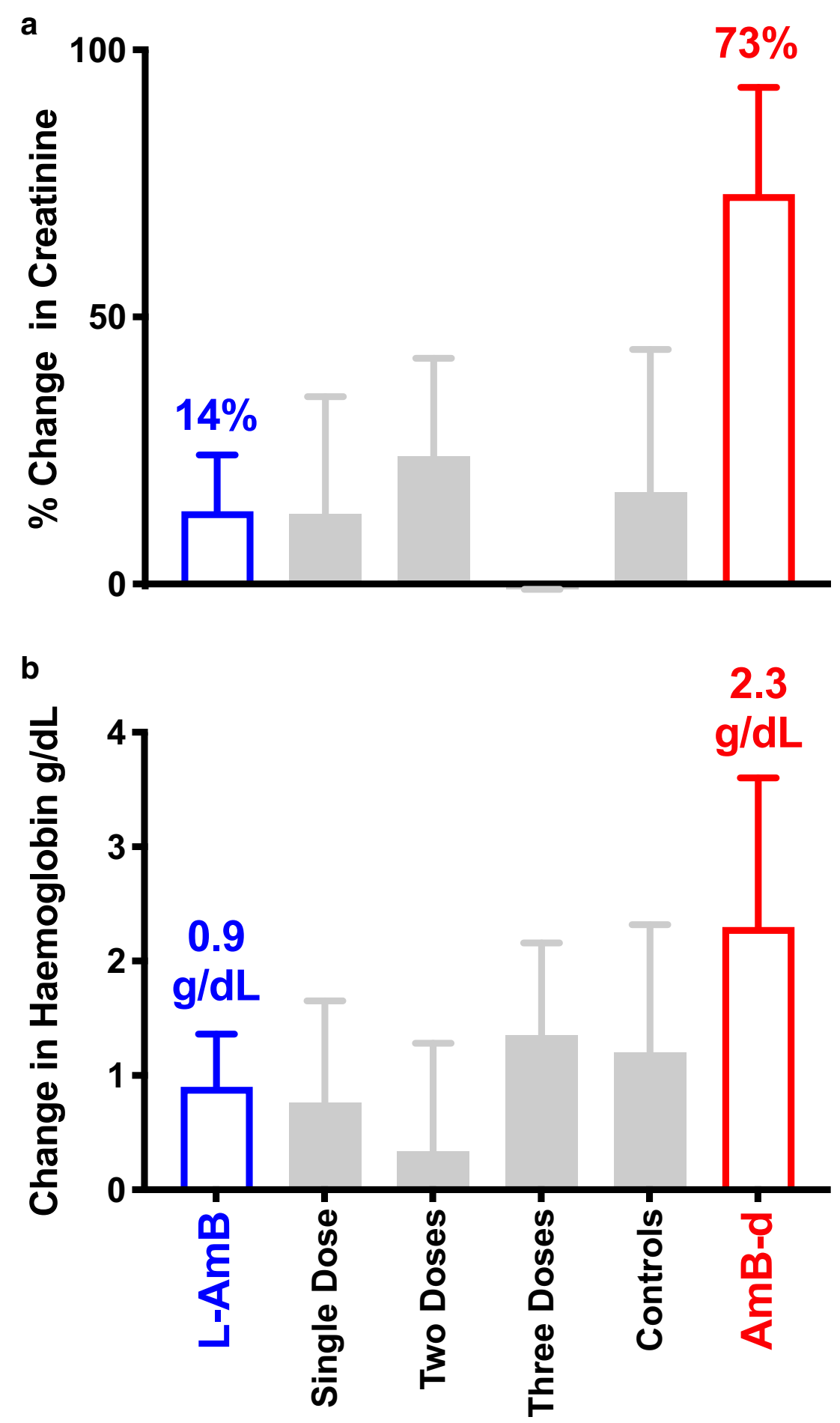

Fig. 5. (a) The median percentage change from baseline creatinine and (b) the median drop in haemoglobin in all treatment arms and each individual treatment arm in the AMBIsome Therapy Induction Optimisation (AMBITION) phase II trial [34••], compared with a pooled analysis of 368 patients treated with amphotericin B [12]. 
commenced recruitment in January 2018 and is expected to complete follow-up in early 2021.

\section{Cryptococcal antigen screening}

Novel short-course and oral therapies for CM may also be relevant for the management of cryptococcal antigenaemia. After individuals with advanced HIV are exposed to Cryptococcus through respiratory inhalation, and usually after a period of dormancy in the lungs, the organism enters the blood stream, causing disseminated cryptococcal disease (cryptococcosis) before then reaching end organs, most commonly the central nervous system [38]. Throughout the disease, Cryptococcus sheds its polysaccharide fungal capsule which can be detected in the blood by highly sensitive and specific cryptococcal antigen (CrAg) lateral flow assays [39, 40]. The prevalence of cryptococcal antigenaemia among individuals with a CD 4 count $<100$ cells $/ \mu \mathrm{L}$ is approximately $6.0-6.5 \%[41,42]$, and among those with a CD4 count 101-200 cells/ $\mu \mathrm{L}$ is approximately $2.0 \%$ [42]. Cryptococcal antigenaemia has consistently been shown to be highly predictive of death, most commonly due to the development of $\mathrm{CM}$, and the detection of $\mathrm{CrAg}$ in the blood provides an opportunity to intervene and give preemptive treatment $[43,44]$. CrAg screening programmes have therefore been adopted in many high HIV prevalence settings. The current recommended initial treatment for cryptococcal antigenaemia is fluconazole $1200 \mathrm{mg} /$ day for 2 weeks [45••], and there is consistent evidence that these programmes reduce the incidence of $\mathrm{CM}$ and related mortality [46].

Despite this, there remains a persistent association between CrAg positivity and death, even among those who receive preemptive fluconazole therapy [47-49]. This may be partially because some of these individuals have symptomatic $\mathrm{CM}$ that has not been adequately investigated or because of subclinical $\mathrm{CM}$ which has been found to occur in $25-40 \%$ of asymptomatic individuals with antigenaemia [50-52]. However, even for a subset of the remaining patients who do not have $\mathrm{CM}$, it is likely that fluconazole is not adequate to prevent the development of CM. Given that these individuals are often well and are managed as outpatients, there may be a potential role for short-course intravenous or oral combination therapy in preventing the development of CM. Randomised controlled data on this subject are needed. A clinical trial exploring the potential role for a single, high dose of L-AmB is currently underway in Uganda [53], and a trial of oral combination therapy is also planned.

\section{Implementation challenges}

Despite the growing evidence to support both the use of flucytosine and liposomal amphotericin in effective short-course and/or oral treatment regimens for the treatment of CM in LMICs, there are significant implementation challenges to overcome before they can be widely used in the regions of the world with the highest burden of HIV-associated CM [54].

Flucytosine was developed in 1957 and is no longer on patent but remains expensive due to monopoly pricing by a limited number of manufacturers [55]. 
A 2-week treatment course in North America costs roughly 25,000 USD [56]. Flucytosine is also manufactured in Europe (Mylan, France) and costs 1.20 USD per tablet, meaning that a two-week treatment for a patient weighing $60 \mathrm{~kg}$ costs 200 USD. An economic model using outcome data from the ACTA trial found that, based on a price of 1.30 USD per tablet, the addition of 2 weeks of flucytosine to fluconazole in settings where fluconazole monotherapy is the mainstay of treatment would cost just 65 USD per life-year saved. Reducing the cost of a single tablet to 0.80 USD and 0.40 USD would see this incremental cost-effectiveness ratio reduce to 44 USD and 28 USD per life-year saved, respectively [57].

Another barrier to the implementation of flucytosine therapy is that until recently, it was not registered in any African countries. This is changing thanks to the efforts of stakeholders in individual countries, the incorporation of flucytosine into national treatment guidelines such as in South Africa [45••], and internationally coordinated initiatives aiming to widen access to flucytosine across countries with a high incidence of CM. In order to keep up with this burgeoning demand, there is also a need to increase manufacturing capacity.

Access to liposomal amphotericin is also impacted by the same economic factors. A single vial costs between 80 USD and 400 USD, and a single high-dose treatment for a patient weighing $60 \mathrm{~kg}$ requires 12 vials [58]. In 2018, the manufacturers, Gilead Sciences, announced that vials of AmBisome would become available at cost price as part of an expanded access programme for CM in 116 LMICs [59], as is already the case for visceral leishmaniasis. Dependent upon the results of the AMBITION trial, an economic evaluation will be conducted to determine the costeffectiveness of giving a single high-dose [58].

\section{Conclusion}

The incidence and mortality associated with HIV-associated cryptococcal meningitis is still unacceptably high, despite widened access to ART. This is in part due to the suboptimal effects of fluconazole monotherapy, significant druginduced toxicities associated with amphotericin $\mathrm{B}$, and limited access to superior treatments. The recently completed ACTA trial has provided evidence to support the use of a short-course amphotericin $\mathrm{B}$ and oral combination antifungal treatment regimens for $\mathrm{CM}$, and the ongoing AMBITION trial hopes to usher in a short-course, high-dose treatment with liposomal amphotericin. Significant implementation challenges must be overcome to ensure that these research findings contribute to a reduction in mortality from this devastating disease.

\section{Authors' contribution}

LRM, JM, and DSL co-wrote the manuscript. JNJ reviewed and approved the final manuscript. 
Funding JNJ is the co-Chief Investigator and DSL is Lead Clinician on the AMBIsome Therapy Induction OptimisatiON (AMBITION) trial which is jointly funded through the European Developing Countries Clinical Trials Partnership (EDCTP), the Swedish International Development Cooperation Agency (SIDA), and the Wellcome Trust/Medical Research Council (UK)/UKAID Joint Global Health Trials. This work was funded by the National Institute for Health Research (NIHR) through a Global Health Research Professorship to JNJ (RP2017-08-ST2-012) using the UK aid from the UK Government to support global health research. The views expressed in this publication are those of the author(s) and not necessarily those of the NIHR or the UK Department of Health and Social Care.Availability of data and materialThe data used to inform this review is available through published articles.Code availabilityNot applicable.

\section{Compliance with ethical standards}

\section{Conflict of interest}

JNJ is the co-Chief Investigator and DSL is the Lead Clinician on the AMBIsome Therapy Induction OptimisatioN (AMBITION) trial. LRM and JM declare that they have no conflicts of interest.

\section{Ethics approval}

Not applicable.

\section{Consent to participate}

Not applicable.

\section{Consent for publication}

Not applicable.

Open Access This article is licensed under a Creative Commons Attribution 4.0 International License, which permits use, sharing, adaptation, distribution and reproduction in any medium or format, as long as you give appropriate credit to the original author(s) and the source, provide a link to the Creative Commons licence, and indicate if changes were made. The images or other third party material in this article are included in the article's Creative Commons licence, unless indicated otherwise in a credit line to the material. If material is not included in the article's Creative Commons licence and your intended use is not permitted by statutory regulation or exceeds the permitted use, you will need to obtain permission directly from the copyright holder. To view a copy of this licence, visit http://creativecommons.org/licenses/by/4.0/.

\section{References and Recommended Reading}

Papers of particular interest, published recently, have been highlighted as:

- Of importance

$\bullet \quad$ Of major importance

1. Jarvis JN, Boulle A, Loyse A, Bicanic T, Rebe K, Williams A, et al. High ongoing burden of cryptococcal dis ease in Africa despite antiretroviral roll out. AIDS. 2009;23:1182-3. https://doi.org/10.1097/QAD. ob013e32832be0fc.

2. Tenforde MW, Mokomane M, Leeme T, Patel RKK, Lekwape N, Ramodimoosi C, et al. Advanced human

immunodeficiency virus disease in Botswana following successful antiretroviral therapy rollout: incidence of and temporal trends in cryptococcal meningitis. Clin Infect Dis. 2017;65:779-86. https://doi.org/10.1093/ $\mathrm{cid} / \mathrm{cix} 430$.

3. Jarvis JN, Meintjes G, Williams A, Brown Y, Crede T, Harrison TS. Adult meningitis in a setting of high HIV 
and TB prevalence: findings from 4961 suspected cases BMC Infect Dis. 2010;10. https://doi.org/10.1186/ 1471-2334-10-67.

4. Rajasingham R, Smith RM, Park BJ, et al. Global burden of disease of HIV-associated cryptococcal meningitis: an updated analysis. Lancet Infect Dis. 2017. https://doi.org/10.1016/S1473-3099(17)30243-8

The most current and comprehensive global burden of disease estimates for HIV-associated cryptococcal meningitis.

5.• Molloy SF, Kanyama C, Heyderman RS, et al. Antifungal combinations for treatment of cryptococcal meningitis in Africa. N Engl J Med. 2018. https://doi.org/10. 1056/NEJMoa1710922.

The ACTA trial demonstrated the non-inferiority of shortcourse (one week) amphotericin B plus flucytosine in the treatment of cryptococcal meningitis as well as the effectiveness of fluconazole plus flucytosine, an oral combination regimen.

6. Longley N, Muzoora C, Taseera K, Mwesigye J, Rwebembera J, Chakera A, et al. Dose response effect of high-dose fluconazole for HIV-associated cryptococcal meningitis in southwestern Uganda. Clin Infect Dis. 2008;47:1556-61. https://doi.org/10.1086/593194.

7. Jackson AT, Nussbaum JC, Phulusa J, Namarika D, Chikasema M, Kanyemba $\mathrm{C}$, et al. A phase II randomized controlled trial adding oral flucytosine to high-dose fluconazole, with short-course amphotericin B, for cryptococcal meningitis. AIDS. 2012;26:1363-70. https://doi.org/10.1097/QAD. ob013e328354b419.

8. Sudan A, Livermore J, Howard SJ, al-Nakeeb Z, Sharp A, Goodwin J, et al. Pharmacokinetics and pharmacodynamics of fluconazole for cryptococcal meningoencephalitis: implications for antifungal therapy and in vitro susceptibility breakpoints. Antimicrob Agents Chemother. 2013;57(6):2793-800. https://doi.org/10 1128/AAC.00216-13.

9. Chesdachai S, Rajasingham R, Nicol MR, Meya DB, Bongomin F, Abassi M, et al. Minimum inhibitory concentration distribution of fluconazole against Cryptococcus species and the fluconazole exposure prediction model. Open Forum Infect Dis. 2019;6. https://doi.org/10.1093/ofid/ofz369.

10. Naicker SD, Mpembe RS, Maphanga TG, Zulu TG, Desanto D, Wadula J, et al. Decreasing fluconazole susceptibility of clinical south african Cryptococcus neoformans isolates over a decade. PLoS Negl Trop Dis. 2020;14(3):1-11. https://doi.org/10.1371/ journal.pntd.0008137.

11. Hope W, Stone NRH, Johnson A, McEntee L, Farrington N, Santoro-Castelazo A, et al. Fluconazole monotherapy is a suboptimal option for initial treatment of cryptococcal meningitis because of emergence of resistance. MBio. 2019;10. https://doi.org/10.1128/ mBio.02575-19.

12. Bicanic T, Bottomley C, Loyse A, Brouwer AE, Muzoora $\mathrm{C}$, Taseera $\mathrm{K}$, et al. Toxicity of amphotericin B deoxycholate-based induction therapy in patients with HIV-associated cryptococcal meningitis. Antimicrob
Agents Chemother. 2015;59:7224-31. https://doi.org/ 10.1128/AAC.01698-15.

13. Patel RKK, Leeme T, Azzo C, et al. High mortality in HIV-associated cryptococcal meningitis patients treated with amphotericin B-based therapy under routine care conditions in Africa. Open Forum Infect Dis. 2018;5(11):ofy267. https://doi.org/10.1093/ofid/ ofy 267.

14. Day JN, Chau TTH, Wolbers M, Mai PP, Dung NT, Mai $\mathrm{NH}$, et al. Combination antifungal therapy for cryptococcal meningitis. N Engl J Med. 2013;368:1291-302. https://doi.org/10.1056/NEJMoa1110404.

15. Rajasingham R, Meya DB, Greene GS, Jordan A, Nakawuka M, Chiller TM, et al. Evaluation of a national cryptococcal antigen screening program for HIVinfected patients in Uganda: a cost-effectiveness modeling analysis. PLoS One. 2019;14:e0210105. https://doi.org/10.1371/journal.pone.0210105.

16. Lawrence DS, Youssouf N, Molloy SF, et al. AMBIsome Therapy Induction OptimisatioN (AMBITION): high dose ambisome for cryptococcal meningitis induction therapy in sub-Saharan Africa: study protocol for a phase 3 randomised controlled non-inferiority trial. Trials. 2018;19(649):649.

17. Jarvis JN, Harrison TS, Lawn SD, Meintjes G, Wood R, Cleary S. Cost effectiveness of cryptococcal antigen screening as a strategy to prevent HIV-associated cryptococcal meningitis in South Africa. PLoS One.

2013;8:e69288. https://doi.org/10.1371/journal.pone. 0069288 .

18. Livermore J, Howard SJ, Sharp AD, et al. Efficacy of an abbreviated induction regimen of amphotericin $\mathrm{B}$ deoxycholate for cryptococcal meningoencephalitis: 3 days of therapy is equivalent to 14 days. MBio. 2014;5(1):1-11. https://doi.org/10.1128/mBio. 00725-13

An animal model study which found no statistically significant difference in cerebral fungal density between rabbits that received daily therapy with amphotericin B and those that received an abbreviated regimen.

19. Muzoora CK, Kabanda T, Ortu G, Ssentamu J, Hearn P, Mwesigye J, et al. Short course amphotericin B with high dose fluconazole for HIV-associated cryptococcal meningitis. J Inf Secur. 2012;64:76-81. https://doi.org/ 10.1016/j.jinf.2011.10.014.

20. Van Der Horst C, Saag M, Cloud G, et al. Treatment of cryptococcal meningitis associated with the acquired immunodeficiency syndrome. N Engl J Med. 1997;337:15-21.

21. Nussbaum JC, Jackson A, Namarika D, Phulusa J, Kenala J, Kanyemba C, et al. Combination flucytosine and high-dose fluconazole compared with fluconazole monotherapy for the treatment of cryptococcal meningitis: a randomized trial in Malawi. Clin Infect Dis. 2010;50:338-44. https://doi.org/10.1086/649861.

22. Tenforde MW, Shapiro AE, Rouse B, et al. Treatment for HIV-associated cryptococcal meningitis. Cochrane Database Syst Rev. 2018;2018(7). doi:https://doi.org/10. 1002/14651858.CD005647.pub3 
23. WHO. World Health Organisation Guidelines for the diagnosis, prevention and management of cryptococcal disease in HIV-infected adults, adolescents and children. Guidel Diagnosis, Prev Manag Cryptococcal Dis HIV-Infected Adults, Adolesc Child Suppl to 2016 Consol Guidel Use Antiretrovir Drugs Treat Prev HIV Infect. 2018.

The WHO guidelines for the management of HIV-associated cryptococcal meningitis which is the current leading international guidance on screening and treatment.

24. Perfect JR, Dismukes WE, Dromer F, et al. The Management of Cryptococcal Disease. IDSA Endorsed. 2010. https://doi.org/10.1086/649858.

25. Nelson M, Dockrell D, Edwards S. British HIV Association and British Infection Association guidelines for the treatment of opportunistic infection in HIVseropositive individuals 2011. HIV Med 2011. doi:https://doi.org/10.1111/j.1468-1293.2011. 00944_1.x, 12, 1, 5.

26. Hamill RJ, Sobel JD, El-Sadr W, et al. Comparison of 2 doses of liposomal amphotericin B and conventional amphotericin B deoxycholate for treatment of AIDSassociated acute cryptococcal meningitis: a randomized, double-blind clinical trial of efficacy and safety. Clin Infect Dis. 2010;51:225-32. https://doi.org/10. 1086/653606.

27. Vogelsinger H, Weiler S, Djanani A, Kountchev J, Bellmann-Weiler R, Wiedermann CJ, et al.

Amphotericin B tissue distribution in autopsy material after treatment with liposomal amphotericin B and amphotericin B colloidal dispersion. J Antimicrob Chemother. 2006;57:1153-60. https://doi.org/10. 1093/jac/dkl141.

28. Lestner J, McEntee L, Johnson A, et al. Experimental models of short courses of liposomal amphotericin b for induction therapy for cryptococcal meningitis. Antimicrob Agents Chemother. 2017. https://doi.org/ 10.1128/AAC.00090-17

A pharmacokinetic study of liposomal amphotericin B in plasma and cerebrum in cohorts of mice infected with Cryptococcus neoformansdemonstrating a prolonged half-life of circa 133 hours and therefore the potential rationale for highdose therapy in humans.

29. O'Connor L, Livermore J, Sharp AD, et al. Pharmacodynamics of liposomal amphotericin $b$ and flucytosine for cryptococcal meningoencephalitis: safe and effective regimens for immunocompromised patients. J Infect Dis. 2013;208:351-61. https://doi.org/10.1093/ infdis/jit164.

30. Cornely OA, Maertens J, Bresnik M, Ebrahimi R, Ullmann AJ, Bouza E, et al. Liposomal amphotericin B as initial therapy for invasive mold infection: a randomized trial comparing a high-loading dose regimen with standard dosing (AmBiLoad Trial). Clin Infect Dis. 2007;44:1289-97. https://doi.org/10.1086/ 514341.

31. Sundar S, Chakravarty J, Agarwal D, Rai M, Murray HW. Single-dose liposomal amphotericin B for visceral leishmaniasis in India. N Engl J Med. 2010;362:50412. https://doi.org/10.1056/NEJMoa0903627.
32. Mehta P, Vinks A, Filipovich A, Vaughn G, Fearing D, Sper C, et al. High-dose weekly amBisome antifungal prophylaxis in pediatric patients undergoing hematopoietic stem cell transplantation: a pharmacokinetic study. Biol Blood Marrow Transplant. 2006;12:23540. https://doi.org/10.1016/j.bbmt.2005.10.010.

33. Giannella M, Ercolani G, Cristini F, Morelli M, Bartoletti M, Bertuzzo V, et al. High-dose weekly liposomal amphotericin B antifungal prophylaxis in patients undergoing liver transplantation: a prospective phase II trial. Transplantation. 2015;99:848-54. https://doi.org/10.1097/TP.0000000000000393.

34 -• Jarvis JN, Leeme TB, Molefi M, et al. Short-course highdose liposomal amphotericin B for human immunodeficiency virus-associated cryptococcal meningitis: a phase 2 randomized controlled trial. Clin Infect Dis. 2019. doi:https://doi.org/10.1093/cid/ciy515.

A phase two trial comparing different high-dose, short-course regimens of liposomal amphotericin B with 14 days of daily dosing. A single, high-dose $(10 \mathrm{mg} / \mathrm{kg})$ was found to be noninferior to daily dosing in terms of clearing Cryptococcus from the cerebrospinal fluid. This regimen has been taken forward to a phase III, clinical-endpoint trial.

35. Beardsley J, Wolbers M, Kibengo FM, Ggayi AB, Kamali A, Cuc NT, et al. Adjunctive dexamethasone in HIVassociated cryptococcal meningitis. $\mathrm{N}$ Engl J Med. 2016;374:542-54. https://doi.org/10.1056/ NEJMoa1509024.

36. Jarvis JN, Bicanic T, Loyse A, Namarika D, Jackson A, Nussbaum JC, et al. Determinants of mortality in a combined cohort of 501 patients with HIV-associated cryptococcal meningitis: implications for improving outcomes. Clin Infect Dis. 2014;58:736-45. https:// doi.org/10.1093/cid/cit794.

37. Boulware DR, Meya DB, Muzoora C, Rolfes MA, Huppler Hullsiek K, Musubire A, et al. Timing of antiretroviral therapy after diagnosis of cryptococcal meningitis. N Engl J Med. 2014;370:2487-98. https://doi. org/10.1056/NEJMoa1312884.

38. Maziarz EK, Perfect JR. Cryptococcosis. Infect Dis Clin N Am. 2016;30:179-206. https://doi.org/10.1016/j. idc.2015.10.006.

39. Jarvis JN, Percival A, Bauman S, Pelfrey J, Meintjes G, Williams GN, et al. Evaluation of a novel point-of-care cryptococcal antigen test on serum, plasma, and urine from patients with HIV-associated cryptococcal meningitis. Clin Infect Dis. 2011;53:1019-23. https://doi. org/10.1093/cid/cir613.

40. Lindsley MD, Mekha N, Baggett HC, Surinthong Y, Autthateinchai R, Sawatwong P, et al. Evaluation of a newly developed lateral flow immunoassay for the diagnosis of cryptococcosis. Clin Infect Dis. 2011;53:321-5. https://doi.org/10.1093/cid/cir379.

41. Temfack E, Bigna JJ, Luma HN, Spijker R, Meintjes G, Jarvis JN, et al. Impact of routine cryptococcal antigen screening and targeted preemptive fluconazole therapy in antiretroviral-naive human immunodeficiency virus-infected adults with CD4 cell counts $<100 / \mu \mathrm{L}$ : a 
systematic review and meta-analysis. Clin Infect Dis. 2019;68:688-98. https://doi.org/10.1093/cid/ciy567.

42. Ford N, Shubber Z, Jarvis JN, Chiller T, Greene G, Migone $\mathrm{C}$, et al. CD4 cell count threshold for cryptococcal antigen screening of HIV-infected individuals: a systematic review and meta-analysis. Clin Infect Dis. 2018;66:S152-9. https://doi.org/10.1093/cid/cix1143.

43. Jarvis JN, Lawn SD, Vogt M, Bangani N, Wood R, Harrison TS. Screening for cryptococcal antigenemia in patients accessing an antiretroviral treatment program in South Africa. Clin Infect Dis. 2009;48:856-62. https://doi.org/10.1086/597262.

44. Liechty CA, Solberg P, Were W, Ekwaru JP, Ransom RL, Weidle PJ, et al. Asymptomatic serum cryptococcal antigenemia and early mortality during antiretroviral therapy in rural Uganda. Tropical Med Int Health. 2007;12:929-35.

45.• Govender NP, Meintjes G, Mangena P, et al. Southern African HIV Clinicians Society guideline for the prevention, diagnosis and management of cryptococcal disease among HIV-infected persons: 2019 update. South Afr J HIV Med. 2019. https://doi.org/10.4102/ sajhivmed.v20i1.1030

This 2019 guidance from the Southern Africa Clinicians Society incorporates the latest research findings and is currently the most up-to-date guidance on the diagnosis and management of cryptococcal meningitis.

46. Mfinanga S, Chanda D, Kivuyo SL, Guinness L, Bottomley C, Simms V, et al. Cryptococcal meningitis screening and community-based early adherence support in people with advanced HIV infection starting antiretroviral therapy in Tanzania and Zambia: an open-label, randomised controlled trial. Lancet. 2015;385:2173-82. https://doi.org/10.1016/S01406736(15)60164-7.

47. Pac L, Horwitz MM, Namutebi AM, Auerbach BJ Semeere A, Namulema T, et al. Implementation and operational research: integrated pre-antiretroviral therapy screening and treatment for tuberculosis and cryptococcal antigenemia. J Acquir Immune Defic Syndr. 2015;68:e69-76. https://doi.org/10.1097/QAI. 0000000000000527.

48. Letang E, Müller MC, Ntamatungiro AJ, Kimera N, Faini D, Furrer H, et al. Cryptococcal antigenemia in immunocompromised human immunodeficiency virus patients in rural Tanzania: a preventable cause of early mortality. Open Forum Infect Dis. 2015;2. https://doi.org/10.1093/ofid/ofv046.

49. Wake RM, Govender NP, Omar T, Nel C, Mazanderani $\mathrm{AH}$, Karat AS, et al. Cryptococcal-related mortality despite fluconazole preemptive treatment in a cryptococcal antigen screen-and-treat program. Clin Infect Dis. 2020;70:1683-90. https://doi.org/10.1093/cid/ ciz485.

50. Longley N, Jarvis JN, Meintjes G, Boulle A, Cross A, Kelly N, et al. Cryptococcal antigen screening in patients initiating ART in South Africa: a prospective cohort study. Clin Infect Dis. 2016;62:581-7. https://doi. org/10.1093/cid/civ936.
51. Wake RM, Britz E, Sriruttan C, Rukasha I, Omar T, Spencer DC, et al. High cryptococcal antigen titers in blood are predictive of subclinical cryptococcal meningitis among human immunodeficiency virusinfected patients. Clin Infect Dis. 2018;66:686-92. https://doi.org/10.1093/cid/cix872.

52. Pongsai $P$, Atamasirikul K, Sungkanuparph S. The role of serum cryptococcal antigen screening for the early diagnosis of cryptococcosis in HIV-infected patients with different ranges of CD4 cell counts. J Inf Secur. 2010;60:4747. https://doi.org/10.1016/j.jinf.2010.03.015.

53. Identifier NCT03945448, Single dose liposomal amphotericin for asymptomatic cryptococcal antigenaemia. ClinicalTrials.gov [Internet]. Bethesda (MD): National Library of Medicine (US). https://doi. org/10.31525/ct1-nct03945448

54. Loyse A, Burry J, Cohn J, Ford N, Chiller T, Ribeiro I, et al. Leave no one behind: response to new evidence and guidelines for the management of cryptococcal meningitis in low-income and middle-income countries. Lancet Infect Dis. 2019;19:e143-7. https://doi. org/10.1016/S1473-3099(18)30493-6.

55. Shroufi A, Govender NP, Meintjes G, Black J, Nel J, Moosa MYS, et al. Time to embrace access programmes for medicines: lessons from the South African flucytosine access programme. Int J Infect Dis. 2020;95:459-61. https://doi.org/10.1016/j.ijid.2020.02.057.

56. Merry M, Boulware DR. Cryptococcal meningitis treatment strategies affected by the explosive cost of flucytosine in the United States: a cost-effectiveness analysis. Clin Infect Dis. 2016;62:1564-8. https://doi. org/10.1093/cid/ciw151.

57. Shiri T, Loyse A, Mwenge L, Chen T, Lakhi S, Chanda D, et al. Addition of flucytosine to fluconazole for the treatment of cryptococcal meningitis in Africa: a multicountry cost-effectiveness analysis. Clin Infect Dis. 2020;70:26-9. https://doi.org/10.1093/cid/ciz163.

58. Ponatshego PL, Lawrence DS, Youssouf N, Molloy SF, Alufandika M, Bango F, et al. AMBIsome Therapy Induction OptimisatioN (AMBITION): high dose AmBisome for cryptococcal meningitis induction therapy in sub-Saharan Africa: economic evaluation protocol for a randomised controlled trial-based equivalence study. BMJ Open. 2019;9:e026288. https://doi. org/10.1136/bmjopen-2018-026288.

59. Gilead. Gilead sciences announces steep discounts for ambisome to treat cryptococcal meningitis in low - and middle-income countries Company Statement, 2018; (September 7). (PRESS RELEASE) Available at: http:// www.gilead.com/news/gilead-sciences-announcessteep-discounts-for-ambisome

\section{Publisher's Note}

Springer Nature remains neutral with regard to jurisdictional claims in published maps and institutional affiliations. 